\title{
AVALIAÇÃO DA ESTRATÉGIA SAÚdE DA FAMÍLIA SOB A ÓTICA DOS PROFISSIONAIS
}

\section{Evaluation of the family health strategy from the professionals' perspective Evaluación de la estrategia de salud familiar bajo la óptica de los profesionales}

\author{
Maria Fernanda Pereira Gomes (OrcID) \\ Universidade Paulista - UNIP - Assis (SP) - Brasil \\ Lislaine Aparecida Fracolli (OrcID) \\ Escola de Enfermagem da Universidade de São Paulo - EEUSP - São Paulo (SP) - Brasil
}

\section{RESUMO}

Objetivo: Avaliar se os atributos essenciais e derivados da Atenção Primária à Saúde (APS) estão presentes na Estratégia Saúde da Família (ESF). Métodos: Pesquisa avaliativa, quantitativa e descritiva, realizada em 21 municípios da região de Presidente Prudente (São Paulo, Brasil), no período de junho a setembro de 2015. Utilizou-se o instrumento Primary Care Assessment Tool (PCATool) para a coleta de dados, que permite avaliar as variáveis: Acesso de Primeiro Contato (acessibilidade e longitudinalidade), Coordenação (integração de cuidados e sistemas de informações) e Integralidade (serviços disponíveis, serviços prestados, orientação familiar e orientação comunitária). A construção dos escores e médias das variáveis realizou-se com o auxílio do Manual do Instrumento de Avaliação da Atenção Primária à Saúde, elaborado pelo Ministério da Saúde. Na análise estatística, construiu-se a média, o desvio padrão, o mínimo e o máximo das variáveis (atributos da APS). Resultados: Participaram 102 profissionais (19 gestores de saúde, 39 médicos e 44 enfermeiros), entre os quais a maioria possuía ensino superior $(98 \% ; n=100)$, menos da metade possuía especialização em Saúde da Família $(37,3 \% ; n=38)$ e curso de capacitação para trabalhar na ESF $(48 \% ; n=49)$. Os atributos essenciais e derivados receberam os respectivos escores: Acesso de Primeiro Contato - Acessibilidade 5,57, Longitudinalidade: 7,71; Coordenação - Integração de cuidados 7,70, Sistemas de Informações 8,74; Integralidade - Serviços disponíveis 8,29, Serviços prestados 8,40, Orientação familiar 8,53, Orientação comunitária 8,12. Conclusão: Segundo a ótica dos profissionais médicos, enfermeiros e gestores, os atributos essenciais e derivados da APS, avaliados na ESF da região, estão presentes.

Descritores: Avaliação em Saúde; Atenção Primária à Saúde; Estratégia Saúde da Família.

\section{ABSTRACT}

Objective: To check if the essential and derivate attributes of Primary Health Care (PHC) are present in the Family Health Strategy (FHS). Methods: This is a quantitative descriptive evaluation research carried out in 21 municipalities in the region of Presidente Prudente (São Paulo, Brazil) from June to September 2015. The Primary Care Assessment Tool (PCATool) was used to collect data as it allows to evaluate the following variables: First Contact Access (access, utilization), Coordination (integrated care, information system) and Comprehensiveness (services available, services provided, family orientation and community orientation). Scores and means of the variables were constructed based on the Manual of the PHC Assessment Tool designed by the Ministry of Health. The statistical analysis calculated the mean, the standard deviation, and the minimum and maximum values of the variables (PHC attributes). Results: Participants were 102 professionals (19 health managers, 39 physicians, and 44 nurses). The majority had higher education (98\%; $n=100)$, less than half specialized in Family Health (37.3\%; $n=38)$ and attended a training course to work in the FHS (48\%; n=49). The essential and derivate attributes obtained the following scores: First Contact Access - Accessibility, 5.57; Utilization, 7.71; Coordination - Integrated Care, 7.70; Coordination - Information Systems, 8.74; Comprehensiveness - Services available, 8.29; Comprehensiveness - Services provided, 8.40; Family orientation, 8.53; Community orientation, 8.12. Conclusion: From the physicians', nurses' and managers' perspective, the essential and derivate attributes of PHC evaluated in the FHS of the region are present.

Descriptors: Health Evaluation; Primary Health Care; Family Health Strategy. 


\section{RESUMEN}

Objetivo: Evaluar si los atributos esenciales y derivados de la Atención Primaria de Salud (APS) se encuentran en la Estrategia de Salud Familiar (ESF). Métodos: Investigación evaluativa, cuantitativa y descriptiva realizada en 21 municipios de la región de Presidente Prudente (São Paulo, Brasil) en el período entre junio y septiembre de 2015. Se utilizó el instrumento Primary Care Assessment Tool (PCATool) para la recogida de datos que permite evaluar las variables: Acceso de Primer Contacto (accesibilidad y longitudinalidad), Coordinación (integración de cuidados y sistemas de informaciones) e Integralidad (servicios disponibles, servicios prestados, orientación familiar y orientación comunitaria). La construcción de las puntuaciones y medias de las variables se dio con el auxilio del Manual del Instrumento de Evaluación de la Atención Primaria de Salud elaborado por el Ministerio de Salud. En el análisis estadístico se construyó la media, la desviación típica, el mínimo y el máximo de las variables (atributos de la APS). Resultados: Participaron 102 profesionales (19 gestores de salud, 39 médicos y 44 enfermeros) entre los cuales la mayoría tenía educación superior (98\%; $n=100)$, menos de la mitad tenía posgrado en Salud Familiar (37,3\%; $n=38)$ y curso de capacitación para trabajar en la ESF $(48 \% ; n=49)$. Los atributos esenciales y los derivados recibieron las respectivas puntuaciones: Acceso de Primer Contacto - Accesibilidad 5,57, Longitudinalidad: 7,71; Coordinación - Integración de cuidados 7,70, Sistemas de Informaciones 8,74; Integralidad - Servicios disponibles 8,29, Servicios prestados 8,40, Orientación familiar 8,53, Orientación comunitaria 8,12. Conclusión: Los atributos esenciales y los derivados de la APS están presentes en la ESF de la región según la óptica de los profesionales médicos, enfermeros y gestores.

Descriptores: Evaluación en Salud; Atención Primaria de Salud; Estrategia de Salud Familiar.

\section{INTRODUÇÃO}

AAtenção Primária à Saúde (APS), denominada no Brasil como Atenção Básica (AB), de acordo com a Política Nacional de Atenção Básica (PNAB), é o contato preferencial dos usuários com os sistemas de saúde. Orientase pelos princípios da universalidade, acessibilidade e coordenação do cuidado; do vínculo e continuidade; da integralidade; da responsabilização; da humanização; da equidade e da participação social( ${ }^{(1)}$.

A AB tem a Saúde da Família como estratégia prioritária para a sua organização de acordo com os preceitos do Sistema Único de Saúde (SUS) ${ }^{(1)}$. Na busca da estruturação de um novo modelo de assistência, o Ministério da Saúde (MS) propôs, em 1994, o Programa de Saúde da Família (PSF), posteriormente denominado de Estratégia Saúde da Família (ESF), com o objetivo de reorganizar a prática assistencial, substituindo o modelo de assistência à saúde centrada no médico e altamente medicalizado por um modelo centrado na família, a partir de sua inserção social, possibilitando entender o processo saúde-doença de forma ampliada e gerando intervenções que busquem mais do que ações curativas ${ }^{(2)}$.

A ESF representou um avanço significativo para a política de saúde brasileira, pois visava a ruptura da concepção usual das práticas de saúde voltadas para o modelo biomédico para uma estratégia inovadora, que objetivava a integração e a organização das atividades em um território definido, a identificação e a resolução de problemas, o mapeamento de risco com vistas à equidade e integralidade, a partir de mudanças radicais no sistema, de maneira articulada e contínua ${ }^{(3)}$.

Nos últimos anos, principalmente no Brasil, a definição operacional da APS tem utilizado como parâmetro quatro atributos essenciais e três características para o funcionamento dos serviços de APS, sendo os atributos essenciais: 1) Acesso de primeiro contato do indivíduo com o sistema de saúde; 2) Longitudinalidade; 3) Integralidade e 4) Coordenação da atenção. As três características, chamadas atributos derivados, que qualificam as ações dos serviços de APS, são: atenção à saúde centrada na família (orientação familiar), orientação comunitária e competência cultural ${ }^{(4,5)}$.

Assim, um serviço de atenção básica dirigido à população geral pode ser considerado provedor de atenção primária quando apresenta os quatro atributos essenciais, aumentando o seu poder de interação com os indivíduos e com a comunidade ao apresentar também os atributos derivados ${ }^{(6)}$.

A identificação rigorosa da presença e da extensão dos atributos citados é fundamental para definir um serviço como realmente orientado para a APS ${ }^{(6)}$. Além disso, a identificação empírica dos atributos permite verificar a associação entre estes e os resultados efetivos da atenção sobre a saúde da população(5). Paralelo ao aumento da cobertura dos serviços de atenção primária no Brasil através da ESF, evidencia-se, nacional e internacionalmente, a crescente associação entre os melhores desfechos de saúde e a maior presença e extensão dos atributos da APS ${ }^{(5)}$.

Nesta perspectiva, a adequada operacionalização da ESF no âmbito da APS favorece o desenvolvimento de ações de promoção da saúde que perpassam pelos princípios da intersetorialidade, intrasetorialidade, integralidade, equidade, participação social, autonomia, empoderamento e territorialidade, e os valores de corresponsabilidade, justiça social, humanização e inclusão social( ${ }^{(7)}$. 
Diante da magnitude da ESF como estratégia estruturante do SUS, realizar a sua avaliação é uma tarefa que necessita de um esforço coletivo contínuo. Devido à amplitude própria da APS, uma proposta específica de avaliação costuma se restringir a uma de suas tantas perspectivas. Assim, identificar dentro do cenário de heterogeneidade que caracteriza a ESF o grau de orientação da APS de cada serviço de saúde ou equipe avaliados permite a produção rigorosa de conhecimento sobre sua efetividade ${ }^{(8)}$.

Neste contexto, a presente pesquisa objetiva avaliar se os atributos essenciais e derivados da Atenção Primária à Saúde estão presentes na Estratégia Saúde da Família.

\section{MÉTODOS}

Trata-se de uma pesquisa avaliativa, quantitativa e descritiva, realizada em vinte e um dos vinte e quatro municípios que pertencem às Comissões Intergestores Regionais (CIR) de Alto Capivari e Alta Sorocabana, da Rede Regional de Atenção à Saúde (RRAS 11) de Presidente Prudente ${ }^{(9)}$, interior do estado de São Paulo, Brasil, no período de junho a setembro de 2015.

No ano de 2015, a CIR de Alta Sorocabana era composta por dezenove municípios: Alfredo Marcondes (4.064 mil habitantes), Álvares Machado (24.482 mil habitantes), Anhumas (3.941 mil habitantes), Caiabu (4.201 mil habitantes), Emilianópolis (3.149 mil habitantes), Estrela do Norte (2.752 mil habitantes), Indiana (4.951 mil habitantes), Martinópolis (25.473 mil habitantes), Narandiba (4.564 mil habitantes), Presidente Bernardes (13.724 mil habitantes), Presidente Prudente (218.960 mil habitantes), Pirapozinho (26.146 mil habitantes), Regente Feijó (19.468 mil habitantes), Ribeirão dos Índios (2.248 mil habitantes), Sandovalina (3.974 mil habitantes), Santo Anastásio (21.059 mil habitantes), Santo Expedito (2.963 mil habitantes), Taciba (6.023 mil habitantes) e Tarabai (7.028 mil habitantes). A CIR de Alto Capivari era formada por cinco municípios: lepê (7.966 mil habitantes), João Ramalho (4.361 mil habitantes), Nantes (2.905 mil habitantes), Quatá (13.501 mil habitantes) e Rancharia (29.732 mil habitantes $)^{(9)}$.

Como critérios de inclusão para a presente pesquisa, os municípios deveriam ter cobertura de ESF igual ou superior a 50\%; as equipes de ESF deveriam ter iniciado o seu funcionamento há 5 anos pelo menos e serem localizadas na área urbana; os profissionais (médicos e enfermeiros) deveriam trabalhar há, no mínimo, 1 ano na ESF; e os gestores de saúde deveriam ter a função de Secretário Municipal de Saúde ou Coordenador da Atenção Básica.

O município de Presidente Prudente não atendeu ao critério de cobertura da ESF (igual ou superior a 50\%) e os secretários municipais de saúde dos municípios de Pirapozinho e Santo Expedito não autorizaram a realização da pesquisa. Portanto, para a amostra da presente pesquisa, considerou-se o universo dos profissionais gestores de saúde, médicos e enfermeiros que trabalhavam na ESF das CIR e que atenderam aos critérios pré-estabelecidos.

As CIR de Alto Capivari e Alta Sorocabana possuíam 73 equipes de ESF em funcionamento no ano de 2015, das quais 62 atendiam aos critérios de funcionamento há 5 anos pelo menos e localizavam-se na área urbana. Do total de 73 médicos, 50 trabalhavam na ESF há mais de um ano. Já do total dos 73 enfermeiros, 53 trabalhavam na ESF há mais de um ano. Assim, participaram da presente pesquisa 19 gestores de saúde, 39 médicos e 44 enfermeiros. Entre os 19 gestores, 14 eram secretários municipais de saúde e 5 eram coordenadores da AB.

A coleta de dados da pesquisa realizou-se por intermédio de um questionário para a caracterização dos profissionais em relação às variáveis (categoria profissional, escolaridade, curso de especialização e curso de capacitação para trabalhar na área da ESF) e por meio do instrumento Primary Care Assessment Tool (PCATool), modificado e validado no Brasil, que mede a presença e a extensão dos atributos essenciais e dos atributos derivados da APS ${ }^{(10)}$, e tem como base o modelo de avaliação da qualidade de serviços de saúde, que se baseia na mensuração de aspectos de estrutura, processo e resultados dos serviços de saúde ${ }^{(11)}$.

Acerca da APS, como dito anteriormente, quatro atributos essenciais para o funcionamento dos serviços de APS são considerados: 1) Acesso de primeiro contato do indivíduo com o sistema de saúde: compreende a acessibilidade e a utilização do serviço de saúde como fonte de cuidado a cada novo problema ou novo episódio de um mesmo problema de saúde; 2) Longitudinalidade: existência de uma fonte continuada de atenção, assim como a sua utilização ao longo do tempo; 3) Integralidade: leque de serviços disponíveis e prestados pelo serviço de atenção primária e 4) Coordenação da atenção: pressupõe alguma forma de continuidade, seja por parte do atendimento pelo mesmo profissional, seja por meio de prontuários médicos ou ambos, além do reconhecimento de problemas abordados em outros serviços e a integração desse cuidado no cuidado global do paciente ${ }^{(5)}$.

Consideraram-se, além dos atributos essenciais, três características chamadas atributos derivados, os quais qualificam as ações dos serviços de APS: 1) Atenção à saúde centrada na família (orientação familiar): avaliação 
das necessidades individuais para a atenção integral, considerando o contexto familiar; 2) Orientação comunitária: reconhecimento por parte do serviço de saúde das necessidades em saúde da comunidade através de dados epidemiológicos e do contato direto com a comunidade e 3) Competência cultural: adaptação do provedor (equipe e profissionais de saúde) às características culturais especiais da população para facilitar a relação e a comunicação(5).

Na presente pesquisa, utilizou-se a versão profissionais do PCATool, validada no Brasil por pesquisadores da Universidade Federal do Rio Grande do Sul (UFRGS), que é composta por 77 itens divididos em 8 componentes da seguinte maneira em relação aos atributos da APS ${ }^{(12)}$ :

1) Acesso de primeiro contato - acessibilidade, constituído por 9 indicadores, que buscam saber sobre a possibilidade de funcionamento e atendimento da ESF nos finais de semana e no período noturno, facilidade de agendar consultas e entrar em contato com os profissionais;

2) Longitudinalidade, constituída por 13 indicadores, que verificam a relação entre os profissionais e os usuários no que tange à comunicação, se os profissionais conhecem a vida e os reais problemas de seus pacientes e se ocorre o estabelecimento de vínculo entre profissional e usuário;

3) Coordenação - integração de cuidados, constituída por 6 indicadores, que investigam se os profissionais têm conhecimento das consultas que seus pacientes fazem com especialistas, se quando encaminham pacientes descrevem a história clínica dos mesmos para os especialistas e se recebem devolutiva da avaliação e do quadro clínico do paciente dos especialistas;

4) Coordenação - sistema de informações, constituída por 3 indicadores, que buscam saber se as informações relativas ao quadro clínico dos pacientes, tratamento e consultas estão organizados e adequadamente registrados e se são de fácil acesso para o usuário;

5) Integralidade - serviços disponíveis, constituída por 22 indicadores, que investigam o rol de serviços disponíveis, tais como: aconselhamento nutricional, imunizações, inserção das famílias em programas sociais, saúde bucal, planejamento familiar, aconselhamento ou tratamento para o uso prejudicial de drogas lícitas ou ilícitas, aconselhamento para problemas de saúde mental, sutura de cortes, aconselhamento e solicitação de teste anti-HIV, identificação de problemas auditivos, identificação de problemas visuais, colocação de tala, remoção de verrugas, exame preventivo para câncer de colo de útero, cuidados pré-natais, remoção de unha encravada, aconselhamento sobre as mudanças que acontecem com o envelhecimento, orientações sobre cuidados no domicílio;

6) Integralidade - serviços prestados, constituído por 15 indicadores, que buscam identificar se os profissionais aconselham sobre alimentação saudável, segurança no lar, uso de cinto de segurança, maneiras de lidar com conflitos de família, prática de exercícios físicos, testes de sangue para verificar os níveis de colesterol, se discutem sobre os medicamentos que o paciente está usando, orientam como guardar arma de fogo com segurança, prevenção de queimaduras, prevenção de quedas, prevenção de osteoporose em mulheres, problemas comuns relativos à menstruação ou menopausa, problemas de comportamento e mudanças do crescimento e desenvolvimento das crianças;

7) Orientação Familiar, constituída por 3 indicadores, que identificam se os profissionais levam em consideração a opinião do paciente e incluem a família na construção do plano terapêutico, e se perguntam sobre as doenças e problemas de saúde que a família possui;

8) Orientação Comunitária, constituído por 6 indicadores, que buscam saber se os profissionais fazem visita, se sabem dos problemas de saúde da comunidade, se ouvem opiniões e ideias da comunidade sobre como melhorar os serviços de saúde e se têm conhecimento sobre a presença de usuários no Conselho Local de Saúde.

O instrumento PCATool apresenta as seguintes respostas para cada um dos itens: Com certeza sim (valor=4), Provavelmente sim (valor=3), Provavelmente não (valor=2), Com certeza não (valor=1) e Não sei/não lembro $(\text { valor }=9)^{(6)}$. O escore de cada atributo é calculado pela média dos valores das respostas dos itens que compõem cada atributo(6).

Escolheu-se a técnica de entrevista para a coleta das informações do questionário e do instrumento, que ocorreu após o contato telefônico, o agendamento prévio com os profissionais e o aval dos secretários municipais de saúde e coordenadores de $A B$ para não atrapalhar o processo de trabalho e o atendimento à população.

Com o auxílio do editor Microsoft Excel for Windows organizaram-se os dados. Realizou-se a construção dos escores e médias dos atributos essenciais e derivados da APS com o auxílio do Manual do Instrumento de Avaliação da APS, elaborado pelo MS, e utilizou-se o software Statistical Package for Social Science (SPSS), versão $16^{(6)}$, para a análise estatística. Para avaliar se os atributos essenciais e derivados da APS estão presentes na ESF, utilizouse como parâmetro a média de $6,6^{(6)}$. Na análise estatística construiu-se a média, o desvio padrão, o mínimo e o máximo das variáveis (atributos da APS). 
A presente pesquisa recebeu aprovação do Comitê de Ética e Pesquisa (CEP) da Escola de Enfermagem da Universidade de São Paulo sob Parecer $n^{\circ}: 1.024 .138$.

\section{RESULTADOS}

Caracterizando os 102 profissionais que participaram da presente pesquisa, 100 (98\%) possuem ensino superior completo, enquanto $2(2 \%)$ possuem ensino médio completo e são gestores de saúde. Em relação à especialização, $38(37,3 \%)$ são especialistas em Saúde da Família. Quanto à participação em curso de capacitação para atuar na ESF, somente $49(48 \%)$ a receberam.

$\mathrm{Na}$ avaliação da presença e da extensão dos atributos essenciais e derivados da APS nas ESFs das CIR de Alto Capivari e Alta Sorocabana, observa-se que os atributos Longitudinalidade, Coordenação - Integração de Cuidados, Coordenação - Sistemas de Informação, Integralidade - Serviços Disponíveis, Integralidade - Serviços Prestados, Orientação Familiar e Orientação Comunitária obtiveram escore acima da média de 6,6(6), exceto o atributo Acesso de Primeiro Contato - Acessibilidade, que obteve escore igual a 5,57. Destaca-se o componente Coordenação - Sistema de Informações, do atributo Coordenação da Atenção, como o melhor avaliado pelos profissionais, apresentando valor igual a 8,74 (Tabela I).

Tabela I - Atributos essenciais e derivados da Atenção Primária à Saúde (APS) segundo os profissionais que trabalham na Estratégia Saúde da Família (ESF). Região de Presidente Prudente, São Paulo, Brasil, 2015.

\begin{tabular}{|c|c|c|c|c|c|}
\hline Atributos & $\mathbf{n}$ & Média & Desvio Padrão & Mínimo & Máximo \\
\hline Acesso de Primeiro Contato - Acessibilidade & 102 & 5,57 & 1,42 & 2,22 & 8,89 \\
\hline Longitudinalidade & 102 & 7,71 & 1,26 & 5,13 & 10,00 \\
\hline Coordenação - Integração de Cuidados & 102 & 7,70 & 1,23 & 4,44 & 10,00 \\
\hline Coordenação - Sistema de informações & 102 & 8,74 & 1,42 & 3,33 & 10,00 \\
\hline Integralidade - Serviços Disponíveis & 102 & 8,29 & 1,24 & 2,42 & 10,00 \\
\hline Integralidade - Serviços Prestados & 102 & 8,40 & 1,57 & 3,11 & 12,44 \\
\hline Orientação Familiar & 102 & 8,53 & 1,74 & 2,22 & 10,00 \\
\hline Orientação Comunitária & 102 & 8,12 & 1,41 & 5,00 & 10,00 \\
\hline Escore Essencial & 102 & 7,73 & 0,85 & 5,01 & 9,58 \\
\hline Escore Geral & 102 & 7,88 & 0,90 & 5,42 & 9,69 \\
\hline
\end{tabular}

$\mathrm{n}$ : número de participantes.

Para avaliar de forma detalhada o componente Acessibilidade, construiu-se a Tabela II, que mostra que mais de $70 \%$ dos profissionais respondeu "Com certeza não" aos indicadores "Seu serviço de saúde está aberto sábado ou domingo?" e "Seu serviço de saúde está aberto, pelo menos em alguns dias da semana, até às 20 horas?". No que tange às questões "Quando seu serviço de saúde está fechado aos sábados e domingos" e "Algum paciente seu fica doente, alguém do seu serviço o atende no mesmo dia?" e "Quando seu serviço de saúde está fechado à noite e algum paciente fica doente, alguém de seu serviço o atende naquela noite?" observou-se que $48,04 \%$ responderam "Com certeza não".

Em relação às questões "Quando seu serviço de saúde está aberto e algum paciente adoece, alguém do seu serviço o atende no mesmo dia?", "Quando o seu serviço de saúde está aberto, os pacientes conseguem aconselhamento rápido pelo telefone quando julgam ser necessário?", "Quando seu serviço de saúde está fechado, existe um número de telefone para o qual os pacientes possam ligar quando adoecem?" e "É fácil para um paciente conseguir marcar hora para uma consulta de revisão de saúde (consulta de rotina, check-up) no seu serviço de saúde?", mais de $60 \%$ dos profissionais responderam "Com certeza sim", confirmando realizar essas ações. Outra informação importante é que 37,25\% dos profissionais responderam "Provavelmente sim" à questão "Na média, os pacientes têm que esperar mais de 30 minutos para serem atendidos pelo médico ou pelo enfermeiro, sem contar a triagem ou o acolhimento?", que se refere ao tempo que o paciente tem que esperar para ser atendido pelo médico ou enfermeiro.

O atributo Longitudinalidade recebeu boa avaliação por parte dos profissionais e os indicadores "No seu serviço de saúde, os pacientes são sempre atendidos pelo mesmo médico/enfermeiro?", "Você consegue entender as perguntas que seus pacientes Ihe fazem?", "Você dá tempo suficiente aos pacientes para falarem sobre as suas preocupações ou problemas?" e "Você acha que seus pacientes se sentem confortáveis ao lhe contar suas preocupações ou problemas?" obtiveram mais de $64 \%$ de respostas "Com certeza sim", indicando que os profissionais 
utilizam a linguagem compressível e dão atenção e tempo suficientes para os pacientes falarem de seus problemas e necessidades de saúde por ser sempre o mesmo médico e enfermeiro que os atende, havendo possibilidade concreta da construção de vínculo.

No indicador "Seus pacientes entendem o que você diz ou pergunta a eles?", $100 \%$ dos participantes responderam "Com certeza sim" e "Provavelmente sim". Mais de 85\% dos participantes responderam "Com certeza sim" e "Provavelmente sim" aos indicadores "Se os pacientes têm uma pergunta, podem telefonar e falar com o médico ou enfermeiro que os conhece melhor?", "Você conhece mais seus pacientes como pessoa do que somente como alguém com um problema de saúde?", "Você entende quais problemas são os mais importantes para os pacientes que você atende?" e "Você teria conhecimento caso seus pacientes não conseguissem as medicações receitadas ou tivessem dificuldades para pagar por elas?", demonstrando que os pacientes conseguem acessar o serviço por intermédio do telefone e que, provavelmente, os profissionais estão utilizando a clínica ampliada.

Em relação às questões "Você sabe quem mora com cada um de seus pacientes?", "Você conhece o histórico de saúde completo de cada paciente?", "Você sabe qual o trabalho ou emprego de cada paciente?" e "Você sabe todos os medicamentos que seus pacientes estão tomando?", observa-se que mais que $20 \%$ dos profissionais responderam "Provavelmente não", indicando que parte significativa dos profissionais não sabe a história clínica, a atividade laboral, os integrantes da família que residem na mesma casa e os medicamentos que os pacientes estão utilizando (Tabela III).

Tabela II - Distribuição percentual das respostas dos profissionais que trabalham na ESF aos itens que compõem o atributo Acesso de Primeiro Contato - Acessibilidade. Região Presidente Prudente, São Paulo, Brasil, 2015.

\begin{tabular}{|c|c|c|c|c|}
\hline $\begin{array}{l}\text { Indicadores do atributo Acesso de } \\
\text { Primeiro Contato - Acessibilidade }\end{array}$ & $\begin{array}{c}\text { Com certeza, } \\
\operatorname{sim} \\
n(\%) \\
\end{array}$ & $\begin{array}{l}\text { Provavelmente } \\
\text { sim } \\
n(\%)\end{array}$ & $\begin{array}{c}\text { Provavelmente } \\
\text { não/não sei/ } \\
\text { não lembro } \\
\text { n (\%) }\end{array}$ & $\begin{array}{c}\text { Com certeza, } \\
\text { não } \\
\text { n (\%) }\end{array}$ \\
\hline Seu serviço de saúde está aberto sábado ou domingo? & $2(1,96)$ & $0(0,00)$ & $9(8,82)$ & $91(89,21)$ \\
\hline $\begin{array}{l}\text { Seu serviço de saúde está aberto, pelo menos em } \\
\text { alguns dias da semana, até às } 20 \mathrm{~h} \text { ? }\end{array}$ & $20(19,61)$ & $4(3,92)$ & $4(3,92)$ & $74(72,55)$ \\
\hline $\begin{array}{l}\text { Quando seu serviço de saúde está aberto e algum } \\
\text { paciente adoece, alguém do seu serviço o atende no } \\
\text { mesmo dia? }\end{array}$ & $79(77,45)$ & $22(21,57)$ & $0(0,00)$ & $1(0,98)$ \\
\hline $\begin{array}{l}\text { Quando o seu serviço de saúde está aberto, os } \\
\text { pacientes conseguem aconselhamento rápido pelo } \\
\text { telefone quando julgam ser necessário? }\end{array}$ & $74(72,55)$ & $24(23,53)$ & $4(3,92)$ & $0(0,00)$ \\
\hline $\begin{array}{l}\text { Quando o seu serviço de saúde está fechado existe um } \\
\text { número de telefone para o qual os pacientes possam } \\
\text { ligar quando adoecem? }\end{array}$ & $63(61,76)$ & $18(17,65)$ & $5(4,90)$ & $16(15,68)$ \\
\hline $\begin{array}{l}\text { Quando o seu serviço de saúde está fechado aos } \\
\text { sábados e domingos e algum paciente seu fica doente, } \\
\text { alguém do seu serviço o atende no mesmo dia? }\end{array}$ & $20(19,61)$ & $11(10,78)$ & $22(21,56)$ & $49(48,04)$ \\
\hline $\begin{array}{l}\text { Quando o seu serviço de saúde está fechado à noite } \\
\text { e algum paciente fica doente, alguém de seu serviço } \\
\text { o atende naquela noite? }\end{array}$ & $21(20,59)$ & $8(7,84)$ & $24(23,53)$ & $49(48,04)$ \\
\hline $\begin{array}{l}\text { É fácil para um paciente conseguir marcar hora para } \\
\text { uma consulta de revisão de saúde (consulta de rotina, } \\
\text { check-up) no seu serviço de saúde? }\end{array}$ & $87(85,29)$ & $13(12,74)$ & $1(0,98)$ & $1(0,98)$ \\
\hline $\begin{array}{l}\text { Na média, os pacientes têm de esperar mais de } 30 \\
\text { minutos para serem atendidos pelo médico ou pelo } \\
\text { enfermeiro, sem contar a triagem ou acolhimento? }\end{array}$ & $19(18,63)$ & $38(37,25)$ & $36(35,29)$ & $9(8,82)$ \\
\hline
\end{tabular}

$\mathrm{n}$ : número de participantes. $\mathrm{h}$ : horas 
Tabela III - Distribuição percentual das respostas dos profissionais que trabalham na ESF aos itens que compõem o atributo Longitudinalidade. Região Presidente Prudente, São Paulo, Brasil, 2015.

\begin{tabular}{|c|c|c|c|c|}
\hline Indicadores do atributo Longitudinalidade & $\begin{array}{l}\text { Com certeza } \\
\text { sim } \\
n(\%)\end{array}$ & $\begin{array}{l}\text { Provavelmente } \\
\text { sim } \\
\mathrm{n}(\%)\end{array}$ & $\begin{array}{l}\text { Provavelmente não/ } \\
\text { não sei/não lembro } \\
\text { n (\%) }\end{array}$ & $\begin{array}{c}\text { Com certeza } \\
\text { não } \\
\text { n (\%) }\end{array}$ \\
\hline $\begin{array}{l}\text { No seu serviço de saúde os pacientes são sempre } \\
\text { atendidos pelo mesmo médico/enfermeiro? }\end{array}$ & $68(66,67)$ & $22(21,57)$ & $8(7,84)$ & $4(3,92)$ \\
\hline $\begin{array}{l}\text { Você consegue entender as perguntas que seus } \\
\text { pacientes Ihe fazem? }\end{array}$ & $80(78,43)$ & $22(21,57)$ & $0(0,00)$ & $0(0,00)$ \\
\hline $\begin{array}{l}\text { Seus pacientes entendem o que você diz ou pergunta } \\
\text { a eles? }\end{array}$ & $60(58,82)$ & $42(41,18)$ & $0(0,00)$ & $0(0,00)$ \\
\hline $\begin{array}{l}\text { Se os pacientes têm uma pergunta, podem telefonar } \\
\text { e falar com o médico ou enfermeiro que os conhece } \\
\text { melhor? }\end{array}$ & $45(44,12)$ & $50(49,02)$ & $5(4,90)$ & $2(1,96)$ \\
\hline $\begin{array}{l}\text { Você dá aos pacientes tempo suficiente para falarem } \\
\text { sobre as suas preocupações ou problemas? }\end{array}$ & $84(82,35)$ & $18(17,65)$ & $0(0,00)$ & $0(0,00)$ \\
\hline $\begin{array}{l}\text { Você acha que seus pacientes se sentem confortáveis } \\
\text { ao lhe contar suas preocupações ou problemas? }\end{array}$ & $66(64,71)$ & $35(34,31)$ & $1(0,98)$ & $0(0,00)$ \\
\hline $\begin{array}{l}\text { Você conhece mais seus pacientes como pessoa } \\
\text { do que somente como alguém com um problema } \\
\text { de saúde? }\end{array}$ & $45(44,12)$ & $46(45,10)$ & $9(8,82)$ & $2(1,96)$ \\
\hline $\begin{array}{l}\text { Você sabe quem mora com cada um de seus } \\
\text { pacientes? }\end{array}$ & $21(20,59)$ & $49(48,04)$ & $26(25,49)$ & $6(5,88)$ \\
\hline $\begin{array}{l}\text { Você entende quais problemas são os mais } \\
\text { importantes para os pacientes que você atende? }\end{array}$ & $56(54,90)$ & $43(42,16)$ & $3(2,94)$ & $0(0,00)$ \\
\hline $\begin{array}{l}\text { Você conhece o histórico de saúde completo de } \\
\text { cada paciente? }\end{array}$ & $31(30,39)$ & $44(43,14)$ & $22(21,57)$ & $5(4,90)$ \\
\hline $\begin{array}{l}\text { Você sabe qual o trabalho ou emprego de cada } \\
\text { paciente? }\end{array}$ & $12(11,76)$ & $45(44,12)$ & $33(32,35)$ & $12(11,76)$ \\
\hline $\begin{array}{l}\text { Você teria conhecimento caso seus pacientes não } \\
\text { conseguissem as medicações receitadas ou tivessem } \\
\text { dificuldades de pagar por elas? }\end{array}$ & $37(36,27)$ & $56(54,90)$ & $8(7,84)$ & $1(0,98)$ \\
\hline $\begin{array}{l}\text { Você sabe todos os medicamentos que seus } \\
\text { pacientes estão tomando? }\end{array}$ & $25(24,51)$ & $45(44,12)$ & $28(27,45)$ & $4(3,92)$ \\
\hline
\end{tabular}

n: número de participantes

O atributo Coordenação - Integração de Cuidados apresentou boa avaliação por parte dos profissionais entrevistados, porém, nos indicadores "Você tem conhecimento de todas as consultas que seus pacientes fizeram com especialistas ou serviços especializados?" e "Você recebe do especialista ou do serviço especializado informações úteis sobre o paciente encaminhado?", mais de $30 \%$ dos profissionais respondeu "Provavelmente não", o que indica que o sistema de referência e contrarreferência precisa melhorar a sua operacionalização na região.

Em relação às questões "Alguém de seu serviço de saúde ajuda o paciente a marcar a consulta encaminhada?" e "Quando seus pacientes são encaminhados, você lhes fornece informação escrita para levar ao especialista ou serviço especializado?", mais de $80 \%$ dos profissionais respondeu "Com certeza sim", enquanto que nas questões "Quando seus pacientes necessitam de um encaminhamento, você discute com os pacientes sobre diferentes serviços onde eles poderiam ser atendidos?" e "Após a consulta com o especialista ou serviço especializado, você fala com seu paciente sobre os resultados desta consulta?" as respostas dos profissionais concentraram-se no "Com certeza sim" e "Provavelmente sim" (Tabela IV). 
A avaliação dos escores médios dos atributos da APS de acordo com as categorias profissionais permite afirmar que os gestores de saúde avaliaram com escores médios menores que os médicos e enfermeiros conforme apresenta a Tabela V. Destaca-se, também, que os médicos atribuíram escores médios maiores nos atributos da APS em relação aos enfermeiros e gestores de saúde. Na Tabela $V$ também se observa que os profissionais gestores, médicos e enfermeiros que participaram da presente pesquisa avaliaram o atributo Acesso de Primeiro Contato Acessibilidade, com escores médios inferiores à média de referência de 6,6.

Tabela IV - Distribuição percentual das respostas dos profissionais que trabalham na ESF aos itens que compõem o atributo Coordenação - Integração de Cuidados. Região Presidente Prudente, São Paulo, Brasil, 2015.

\begin{tabular}{|c|c|c|c|c|}
\hline $\begin{array}{l}\text { Indicadores do atributo Coordenação - } \\
\text { Integração de Cuidados }\end{array}$ & $\begin{array}{l}\text { Com } \\
\text { certeza sim } \\
\text { n (\%) }\end{array}$ & $\begin{array}{l}\text { Provavelmente } \\
\text { sim } \\
n(\%)\end{array}$ & $\begin{array}{l}\text { Provavelmente não/ } \\
\text { não sei/ não lembro } \\
\text { n(\%) }\end{array}$ & $\begin{array}{c}\text { Com certeza } \\
\text { não } \\
\text { n (\%) }\end{array}$ \\
\hline $\begin{array}{l}\text { Você tem conhecimento de todas as consultas que } \\
\text { seus pacientes fazem a especialistas ou serviços } \\
\text { especializados? }\end{array}$ & $20(19,61)$ & $47(46,08)$ & $32(31,37)$ & $3(2,94)$ \\
\hline $\begin{array}{l}\text { Quando seus pacientes necessitam de um } \\
\text { encaminhamento, você discute sobre os diferentes } \\
\text { serviços onde eles poderiam ser atendidos? }\end{array}$ & $52(50,98)$ & $46(45,10)$ & $4(3,92)$ & $0(0,00)$ \\
\hline $\begin{array}{l}\text { Alguém de seu serviço de saúde ajuda o paciente } \\
\text { a marcar a consulta encaminhada? }\end{array}$ & $82(80,39)$ & $19(18,63)$ & $1(0,98)$ & $0(0,00)$ \\
\hline $\begin{array}{l}\text { Quando seus pacientes são encaminhados, você } \\
\text { Ihes fornece informação escrita para levar ao } \\
\text { especialista ou serviço especializado? }\end{array}$ & $86(84,31)$ & $14(13,73)$ & $2(1,96)$ & $0(0,00)$ \\
\hline $\begin{array}{l}\text { Você recebe do especialista ou do serviço } \\
\text { especializado informações úteis sobre o paciente } \\
\text { encaminhado? }\end{array}$ & $14(13,73)$ & $51(50,00)$ & $31(30,39)$ & $6(5,88)$ \\
\hline $\begin{array}{l}\text { Após a consulta com o especialista ou serviço } \\
\text { especializado, você fala com seu paciente sobre } \\
\text { os resultados desta consulta? }\end{array}$ & $35(34,31)$ & $58(56,86)$ & $8(7,84)$ & $1(0,98)$ \\
\hline
\end{tabular}

n: número de participantes.

Tabela V - Descrição da pontuação dos escores* médios dos atributos essenciais e derivados de acordo com os profissionais (gestores, médicos e enfermeiros) que trabalham na Estratégia Saúde da Família (ESF). Região Presidente Prudente, São Paulo, Brasil, 2015.

\begin{tabular}{lcccc}
\hline Atributos da APS & $\begin{array}{c}\text { Gestores } \\
\mathbf{n}=\mathbf{1 9}\end{array}$ & $\begin{array}{c}\text { Médicos } \\
\mathbf{n = 3 9}\end{array}$ & $\begin{array}{c}\text { Enfermeiros } \\
\mathbf{n = 4 4}\end{array}$ & $\begin{array}{c}\text { Desvio } \\
\text { padrão }\end{array}$ \\
\hline Acesso de Primeiro Contato - Acessibilidade & 6,07 & 5,43 & 5,50 & 0,29 \\
Longitudinalidade & 6,98 & 8,11 & 7,67 & 0,47 \\
Coordenação - Integração de Cuidados & 7,37 & 8,22 & 7,40 & 0,39 \\
Coordenação - Sistema de Informaçães & 7,84 & 9,32 & 8,64 & 0,60 \\
Integralidade - Serviços Disponíveis & 7,92 & 8,43 & 8,10 & 0,21 \\
Integralidade - Serviços Prestados & 7,17 & 8,61 & 8,75 & 0,71 \\
Orientação Familiar & 7,37 & 9,09 & 8,54 & 0,72 \\
Orientação Comunitária & 7,51 & 8,30 & 8,10 & 0,34 \\
Escore Derivado & 7,44 & 8,70 & 8,32 & 0,33 \\
Escore Essencial & 7,23 & 8,02 & 7,68 & 0,53 \\
Escore Geral & 7,28 & 8,19 & 7,84 & 0,37 \\
\hline
\end{tabular}

*Média de referência de 6,6. APS: Atenção Primária à Saúde 


\section{DISCUSSÃO}

Os resultados do presente estudo apontam que a Acessibilidade, componente do atributo Acesso de Primeiro Contato, não obteve avaliação satisfatória pelos profissionais, pois os valores obtidos são inferiores a média de 6,6, considerada parâmetro em outras pesquisas ${ }^{(13-24)}$.

A acessibilidade pode ser caracterizada pelo que facilita e impede as pessoas de serem atendidas em determinado serviço de saúde ${ }^{(24)}$. Para tanto, a acessibilidade possui quatro componentes: a) Geográfico, refere-se a aspectos físicos impeditivos ao acesso (rios, grandes avenidas) e distância entre a população e os recursos; b) Organizacional, refere-se a obstáculos originados no modo de organização do serviço (facilidade de marcar consultas, horários de atendimento, existência de filas, demora na espera pelo atendimento médico); c) Sociocultural, refere-se às perspectivas da população (percepção do indivíduo sobre a gravidade de sua doença, medo do diagnóstico e das intervenções, crenças, hábitos); d) Econômico, refere-se ao consumo de tempo, energia e recursos financeiros para obtenção da assistência( ${ }^{(24,25)}$.

Mudanças para melhorar a acessibilidade na APS passam por decisão política e administrativa, indo além de modificações no horário de funcionamento e extensão do atendimento da ESF para os finais de semana ${ }^{(13)}$. Para favorecer a ampliação do acesso, deve haver condições para melhor execução do trabalho pela equipe de saúde, o que inclui: profissionais devidamente capacitados; dimensionamento e quantitativo adequado de profissionais; remuneração condizente com os horários e a jornada de trabalho; implantação de protocolos para ampliação do acesso; organização da programação dos serviços; planejamento das ações a serem realizadas, e insumos adequados e disponíveis ${ }^{(13)}$.

Os profissionais entrevistados na presente pesquisa avaliaram de forma satisfatória o atributo Longitudinalidade, assim como em outras pesquisas ${ }^{(13-15,21,26)}$. Entretanto, em pesquisas realizadas nos municípios de Chapecó (Santa Catarina), Piracicaba (São Paulo) e Lajeado (Rio Grande do Sul), os profissionais avaliaram o atributo Longitudinalidade com médias inferiores a 6,6 $6^{(16-18)}$.

Com a avaliação dos itens que compõem o atributo Longitudinalidade nas CIR de Alto Capivari e Alta Sorocabana, é possível afirmar que mais de $25 \%$ dos profissionais responderam não saber da atividade laboral e dos dados socioeconômicos dos pacientes. No entanto, o que chama mais atenção é que $27,45 \%$ desses profissionais relatam que provavelmente não sabem os medicamentos que os pacientes fazem uso. Nesse caso, apesar do atributo Longitudinalidade ter obtido boa avaliação pelos profissionais que participaram da presente pesquisa, indicadores importantes que compõem o atributo em questão obtiveram valores baixos, indicando que existe problemas na continuidade do cuidado ${ }^{(13)}$.

As dificuldades para o estabelecimento de vínculo, a continuidade do cuidado e o conhecimento dos principais problemas apresentados pelas famílias, seja no plano singular, seja coletivo, podem estar sendo fortalecidas pela falta de capacitação dos profissionais para trabalharem na ESF, confirmada pelo resultado de que somente $48 \%$ dos profissionais que participaram da presente pesquisa realizaram curso de capacitação e declararam rotatividade dos profissionais, inexistência de plano de carreira, má remuneração e condições de trabalho precárias ${ }^{(13)}$.

A integração de cuidados está ocorrendo na região segundo os profissionais entrevistados na presente pesquisa, o que está de acordo com o encontrado em pesquisas realizadas em outros cenários brasileiros ${ }^{(13-16,19,21,26,27)}$. Contudo, identificou-se que os profissionais entrevistados no presente estudo declararam desconhecer o caminho percorrido pelos seus pacientes no interior da rede de serviços de saúde. Isto indica que o sistema de referência e contrarreferência nas CIR é frágil, desconhecido pelos profissionais da ESF e que precisa ser aprimorado. $O$ sistema de referência e contrarreferência no âmbito das Redes de Atenção à Saúde (RAS) constitui-se nos fluxos e contrafluxos de informações, produtos e pessoas ao longo dos pontos de atenção de forma dinâmica e contínua( ${ }^{(28)}$.

Na dimensão da Coordenação - Sistema de Informações, observa-se que os profissionais entrevistados na atual pesquisa a avaliaram positivamente, da mesma forma que em outras pesquisas realizadas nos estados de Minas Gerais, Rio Grande do Sul, Santa Catarina, Paraná, Mato Grosso e no Distrito Federal ${ }^{(13,15,16,19,21,26)}$. Apesar dos resultados positivos da presente pesquisa para os componentes do atributo Coordenação da Atenção, Integração de Cuidados e Sistema de Informações, que obtiveram avaliação acima da média de 6,6, observa-se, na análise detalhada de seus itens, que o sistema de referência e contrarreferência é falho.

É reconhecido que o sistema de referência e contrarreferência brasileiro encontra muitos entraves comunicacionais, principalmente pela inexistência, na maioria dos municípios brasileiros, do sistema de prontuário eletrônico integrado entre os níveis primário, secundário e terciário(28). 
O componente Serviços Disponíveis, do atributo Integralidade, obteve média satisfatória nas CIR avaliadas no presente estudo, bem como em outras pesquisas ${ }^{(13-14,16,19)}$. No entanto, em pesquisas realizadas em Cuiabá (Mato Grosso) e no Distrito Federal, a Integralidade - Serviços Disponíveis obteve avaliação insatisfatória ${ }^{(21,26)}$.

$\mathrm{Na}$ avaliação detalhada do componente Integralidade - Serviços Disponíveis da presente pesquisa identificou-se que os procedimentos de colocação de tala, remoção de verruga e remoção de unha encravada não são realizados por boa parte dos profissionais da ESF das CIR de Alto Capivari e Alta Sorocabana. Nas pesquisas realizadas em Belo Horizonte (Minas Gerais) e em Florianópolis (Santa Catarina), a maioria dos médicos e enfermeiros afirma que as pequenas cirurgias não são realizadas na ESF, corroborando com os resultados da presente pesquisa(29,30).

Mudanças nas práticas tradicionais de saúde, as quais pouco respondem ou resolvem os problemas de saúde contemporâneos, só serão construídas a partir de profundas transformações políticas, administrativas e de gestão do processo de trabalho da ESF ${ }^{(13)}$. A lógica dos antigos centros de saúde, modelo tradicional de APS, está tão arraigada nos profissionais e população brasileira que a ESF, embora tenha impactado positivamente nos indicadores de saúde, ainda traz em seu processo de trabalho práticas descontextualizadas dos problemas reais da população e protocolos generalizados, modelo pouco resolutivo quando se trata da APS ${ }^{(13)}$. Nesta conjuntura, é necessário agregar práticas inovadoras no cotidiano dos profissionais de saúde, a fim de responder às novas demandas e necessidades de saúde apresentadas pelas famílias atendidas ${ }^{(13)}$.

O atributo Integralidade - Serviços Prestados obteve avaliação satisfatória dos profissionais que participaram da presente pesquisa, bem como dos profissionais que participaram de pesquisas realizadas em Minas Gerais, Rio de Janeiro (Rio de Janeiro), Porto Alegre (Rio Grande do Sul), Chapecó (Santa Catarina), Cuiabá (Mato Grosso), em municípios de pequeno porte da Região Norte e Nordeste do estado do Rio Grande do Sul e no Distrito Federal(13-16,21,26.). Contudo, as orientações relativas à prevenção de acidentes por arma de fogo não são realizadas pelos profissionais que trabalham na ESF das CIR de Alto Capivari e Alta Sorocabana do presente estudo.

Para tanto, considerando que a integralidade parte do pressuposto da integração dos diferentes níveis assistenciais e que sua operacionalização depende da oferta e da utilização dos serviços em seus diferentes tipos de atendimento, de acordo com as necessidades da população, o MS define a integralidade como a prestação, pela equipe de saúde, de um conjunto de serviços que atendam às necessidades da população adscritas nos campos da promoção, da prevenção, da cura, da reabilitação e da paliação, responsabilizando-se pela oferta de serviços em outros pontos de atenção à saúde e o reconhecimento adequado dos problemas biológicos, psicológicos e sociais que causam as doenças ${ }^{(31)}$.

Nessa conjuntura, faz-se necessário pensar maneiras de melhorar a operacionalização e a disponibilização de serviços no âmbito da ESF ou de organizar o sistema de forma que os serviços que não são disponibilizados pela ESF sejam disponibilizados por outros níveis assistenciais, garantindo atendimento integral ${ }^{(28)}$.

O atributo Orientação Familiar obteve avaliação satisfatória dos profissionais que participaram da presente pesquisa e dos profissionais que participaram de pesquisas realizadas em outros estados ${ }^{(13,15,16,19,21,26)}$. A implantação da ESF visa reorganizar, fortalecer e qualificar a APS no Brasil, mediante mudanças nas práticas tradicionais de cunho curativo e individualizado, para práticas com enfoque na família, comunidade e promoção da saúde ${ }^{(1)}$. Nesse contexto, é imprescindível que os profissionais adotem tais práticas em seu processo de trabalho. Assim, a Orientação Familiar ocorre quando o alcance da integralidade fornece um olhar por parte dos profissionais das potencialidades e fragilidades do contexto familiar dos indivíduos frente ao processo saúde-doença ${ }^{(32)}$.

O atributo Orientação Comunitária obteve avaliação satisfatória dos profissionais das CIR no presente estudo, assim como em outras pesquisas ${ }^{(13-16)}$. A avaliação detalhada do atributo Orientação Comunitária indica que mais de $20 \%$ dos profissionais que trabalham na ESF da região de Presidente Prudente não conhecem os problemas e as necessidades de saúde da população. A operacionalização da orientação comunitária no âmbito da ESF permite o reconhecimento das necessidades das famílias em função do contexto físico, econômico e social em que vivem, e facilita a análise situacional dessas necessidades numa perspectiva populacional para a integração em programas intersetoriais de enfrentamento dos determinantes sociais da saúde ${ }^{(33)}$.

Para a construção de uma APS resolutiva diante dos atuais problemas de saúde pública no Brasil, destacandose as doenças crônicas não transmissíveis como desafio atual para a atuação da ESF, sendo imprescindível a incorporação de práticas de saúde contextualizadas com o campo da promoção da saúde ${ }^{(7)}$. Nesta perspectiva, a incorporação de ações de promoção da saúde no âmbito da ESF é bem-sucedida quando os atributos essenciais e derivados estão presentes e adequadamente operacionalizados ${ }^{(13)}$.

Nessa conjuntura, faz-se necessário apontar que a ESF possui características próprias, como mapeamento de risco, estabelecimento do vínculo com os usuários, conhecimento das relações sociais e familiares da população, 
e proximidade com os reais problemas vivenciados na comunidade, que facilitam o delineamento de ações de promoção da saúde no âmbito da unidade básica de saúde e nos domicílios ${ }^{(1)}$. Embora a Política Nacional de Promoção da Saúde tenha caráter transversal, é na APS que ela tem maior possibilidade de operacionalização, já que as características do processo de trabalho contribuem para sua concretude ${ }^{(7)}$.

Este estudo, em particular, apresenta a limitação de as entrevistas dirigidas aos profissionais concentraram-se em uma área geográfica específica e delimitada, não retratando a avaliação de todos os profissionais que trabalham na ESF.

\section{CONCLUSÃO}

Os atributos essenciais (acesso de primeiro contato do indivíduo com o sistema de saúde, longitudinalidade, integralidade e coordenação da atenção) e derivados (atenção à saúde centrada na família, orientação comunitária e competência cultural) da Atenção Primária à Saúde avaliados na Estratégia Saúde da Família da região, segundo a ótica dos profissionais médicos, enfermeiros e gestores, estão presentes.

O manuscrito foi baseado na tese de doutorado: Avaliação dos Atributos da Atenção Primária à Saúde na Estratégia Saúde da Família das Comissões Intergestores Regionais de Alto Capivari e Alta Sorocabana - SP, da Escola de Enfermagem da Universidade de São Paulo (EEUSP), 2016, 236 p.

\section{CONTRIBUIÇÕES}

Maria Fernanda Pereira Gomes participou do delineamento do estudo, análise e interpretação dos dados e redação final do manuscrito. Lislaine Aparecida Fracolli participou da análise e interpretação dos dados e redação final do manuscrito.

\section{REFERENNCIAS}

1. Brasil. Ministério da Saúde. Portaria $n^{\circ} 2.436$, de 21 de setembro de 2017. Aprova a Política Nacional de Atenção Básica, estabelecendo a revisão de diretrizes para a organização da Atenção Básica, no âmbito do Sistema Único de Saúde (SUS) [acesso em 2017 Jul 21]. Disponível em: http://bvsms.saude.gov.br/bvs/saudelegis/ gm/2017/prt2436_22_09_2017.html

2. Franco T, Merhy E. PSF: contradições e novos desafios. In: $10^{\mathrm{a}}$ Conferência Nacional de Saúde Online; 1996; Brasília. Brasília: Ministério da Saúde; 1996 [acesso em 2017 Jul 21]. Disponível em: http://www.uff.br/ saudecoletiva/professores/merhy/artigos-17.pdf.

3. Soratto J, Pires DEP, Dornelles S, Lorenzetti J. Estratégia saúde da família: uma inovação tecnológica em saúde. Texto \& Contexto Enferm. 2015;24(2):584-92.

4. Starfield B. Primary care: concept, evaluation and policy. New York: Oxford University Press; 1992.

5. Starfield B, Xu J, Shi L. Validating the adult primary care assessment tool. J Fam Pract. 2001;50(2):161-75.

6. Ministério da Saúde (BR), Secretaria de Atenção em Saúde, Departamento de Atenção Básica. Manual do Instrumento de avaliação da atenção primária à saúde: primary care assessment tool pcatool. Brasília: Ministério da Saúde; 2010.

7. Ministério da Saúde (BR), Secretaria de Vigilância em Saúde, Secretaria de Atenção à Saúde. Política Nacional de Promoção da Saúde: revisão da Portaria MS/GM nº 687, de 30 de março de 2006. Brasília: Ministério da Saúde; 2015.

8. Ministério da Saúde (BR), Secretaria de Atenção à Saúde, Departamento da Atenção Básica, Coordenação de Acompanhamento e Avaliação. Avaliação da Atenção Básica em Saúde: caminhos da institucionalização. Brasília: Ministério da Saúde; 2005.

9. Ministério da Saúde (BR). Sala de Apoio a Gestão Estratégica (SAGE) do Ministério da Saúde [Internet]. Brasília: Ministério da Saúde; 2017. [acesso em 2017 Jul 21]. Disponível em: http://189.28.128.178/sage/

10. Cassady CE, Starfield B, Hurtado MP, Berk RA, Nanda JP, Friedenberg LA. Measuring consumer experiences 
with primary care. Pediatrics. 2000;105(4 Pt 2):998-1003.

11. Donabedian A. Evaluating the quality of medical care. Milbank Q. 2005;83(4):691-729.

12. Hauser L, Castro RCL, Vigo A, Trindade TG, Gonçalves MR, Stein AT, et al. Tradução, adaptação, validade e medidas de fidedignidade do instrumento de avaliação da atenção primária à saúde (PCATool) no Brasil: versão profissionais de saúde. Rev Bras Med Farm Comunidade. 2013;8(29):244-55.

13. Silva SA. Avaliação dos atributos da atenção primária à saúde na estratégia saúde da família em municípios do sul de minas gerais [tese]. São Paulo: Universidade de São Paulo; 2014.

14. Harzheim E, Lima KM, Hauser L. Pesquisa avaliativa sobre aspectos de implantação, estrutura, processo e resultados das Clínicas da Família na cidade do Rio de Janeiro. Porto Alegre: OPAS; 2013.

15. Castro RCL, Knauth DR, Harzheim E, Hauser L, Duncan BB. Avaliação da qualidade da atenção primária pelos profissionais de saúde: comparação entre diferentes tipos de serviços. Cad Saúde Pública. 2012;28(9):1772-84.

16. Vitoria AM, Harzheim E, Takeda SP, Hauser L. Avaliação dos atributos da atenção primária à saúde em Chapecó, Brasil. Rev Bras Med Fam Comunidade. 2013;8(29):285-93.

17. Cesar MC, Campos GWS, Montebelo MIL, Sarmento G. Avaliação da atenção primária no município de Piracicaba, SP, Brasil. Saúde Debate. 2014; 38(Nesp):296-306.

18. Penso JM, Périco E, Oliveira MMC, Strohschoen AAG, Carreno I, Rempela C. Avaliação da Atenção Primária à Saúde utilizando o Instrumento PCATool-Brasil. Rev Bras Med Fam Comunidade. 2017;12(39):1-9.

19. Chomatas E, Vigo A, Marty I, Hauser L, Harzheim E. Avaliação da presença e extensão dos atributos da atenção primária em Curitiba. Rev Bras Med Fam Comunidade. 2013;8(29):294-303.

20. Reis RS, Coimbra LC, Silva AAM, Santos AM, Alves MTSSB, Lamy ZC, et al. Acesso e utilização dos serviços na Estratégia Saúde da Família na perspectiva dos gestores, profissionais e usuários. Ciênc Saúde Colet. 2013;18(11):3321-31.

21. Dias KAPL. Assistência em saúde: uma visão dos usuários e profissionais da atenção básica - Cuiabá - MT [dissertação]. Recife: Centro de Pesquisas Aggeu Magalhães, Fundação Oswaldo Cruz; 2013.

22. Lima EFA, Sousa AI, Leite FMC, Lima RCD, Nascimento MH, Primo CC. Avaliação da Estratégia Saúde da Família na perspectiva dos profissionais de saúde. Esc Anna Nery Rev Enferm. 2016;20(2):275-80.

23. Oliveira, MPR, Menezes IHCF, Sousa LM, Peixoto MRG. formação e qualificação de profissionais de saúde: fatores associados à qualidade da atenção primária. Rev Bras Educ Med. 2016;40(4):547-59.

24. Azevedo ALM, Costa AM. A estreita porta de entrada do Sistema Único de Saúde (SUS): uma avaliação do acesso na Estratégia de Saúde da Família. Interface Comum Saúde Educ. 2010;14(35):797-810.

25. Fekete MC. Estudo de acessibilidade na avaliação dos serviços de saúde. In: Santana JP, organizador. Desenvolvimento gerencial de unidades básicas do Sistema Único de Saúde (SUS). Brasília: OPS; 1997. p. 5-7.

26. Araújo RL, Mendonça AVM, Sousa MA. Percepção dos usuários e profissionais de saúde no Distrito Federal: os atributos da atenção primária. Saúde Debate. 2015;39(105):387-99.

27. Leandro SS, Monteiro PS. Avaliação da coordenação da atenção na perspectiva dos profissionais de saúde. Enferm Foco (Brasília). 2017;8(3):65-9.

28. Mendes EV. A construção social da Atenção Primária à Saúde. Brasília: Conselho Nacional de Secretários de Saúde; 2015.

29. Giovanella L, Escorel S, Mendonça MHM. Estudos de caso sobre implementação da estratégia de saúde da família em quatro grandes centros urbanos: Belo Horizonte. Rio de Janeiro; 2009.

30. Giovanella L, Escorel S, Mendonça MHM, coordenadoras. Estudos de caso sobre a implementação da Estratégia Saúde da Família em grandes centros urbanos: relatório final. Florianópolis. Rio de Janeiro; FIOCRUZ; 2009.

31. Conselho Nacional de Secretários de Saúde (BR). A atenção primária e as redes de atenção à saúde. Brasília: Ministério da Saúde; 2015. 
32. Starfield B. Atenção primaria: equilíbrio entre a necessidade de saúde, serviços e tecnologias. Brasília: Ministério da Saúde; 2002.

33. Mendes EV. O cuidado das condições crônicas na atenção primária à saúde: o imperativo da consolidação da estratégia da saúde da família. Brasília: Organização Pan-Americana da Saúde; 2012.

\section{Endereço para correspondência:}

Maria Fernanda Pereira Gomes

Universidade Paulista - UNIP

Rua Myrthes Spera Conceição, 301

Bairro: Conjunto Nelson Marcondes

CEP: 19813-550 - Assis - SP - Brasil

E-mail: mferpg@usp.br 